\title{
Modular specification of real-time systems
}

\author{
Inal, Recep
}

Published in:

Proceedings of the Sixth Euromicro Workshop on Real-Time Systems

Link to article, DOI:

10.1109/EMWRTS.1994.336871

Publication date:

1994

Document Version

Publisher's PDF, also known as Version of record

Link back to DTU Orbit

Citation (APA):

Inal, R. (1994). Modular specification of real-time systems. In Proceedings of the Sixth Euromicro Workshop on Real-Time Systems (pp. 16-21). IEEE. https://doi.org/10.1109/EMWRTS.1994.336871

\section{General rights}

Copyright and moral rights for the publications made accessible in the public portal are retained by the authors and/or other copyright owners and it is a condition of accessing publications that users recognise and abide by the legal requirements associated with these rights.

- Users may download and print one copy of any publication from the public portal for the purpose of private study or research.

- You may not further distribute the material or use it for any profit-making activity or commercial gain

- You may freely distribute the URL identifying the publication in the public portal

If you believe that this document breaches copyright please contact us providing details, and we will remove access to the work immediately and investigate your claim. 


\title{
Modular Specification of Real-Time Systems *
}

\author{
Recep Inal \\ Department of Computer Science \\ Technical University of Denmark, bldg. 344 \\ DK 2800 Lyngby, Denmark \\ Email: rei@id.dth.dk
}

\begin{abstract}
Duration Calculus, a real-time interval logic, has been embedded in the $Z$ specification language to provide a notation for real-time systems that combines the modularisation and abstraction facilities of $Z$ with a logic suitable for reasoning about real-time properties [4, 2]. In this article the notation is presented through a top-level specification of requirements for a simple Air Traffic Monitoring system, and reasoning is illustrated by a refinement towards a design.
\end{abstract}

Keywords: real-time systems, requirements, formal specifications, refinement, design.

\section{Introduction}

A purpose of formal specifications is to contribute to concise and understandable documentation of requirements, assumptions and design. Furthermore they shall allow a designer to reason about correctness of a design with respect to requirements under given assumptions. Previous work within the Provably Correct Systems project [1] has demonstrated use of Duration Calculus, a real-time interval logic as a notation for concise specification and reasoning about real-time systems $[9,3,5]$. In that work, the notation is used in the usual mathematical fashion with implicit declarations and modularisation through textual subdivision. This paper illustrates an application of a new development, where the Duration Calculus is embedded in the widely used $Z$ language [11] which provides a precise explicit notation for modular specifications suitable for larger scale developments.

The timed $\mathrm{Z}$ notation is illustrated by means of a case study which is based on an air traffic monitoring problem posed by the University of Stirling[7]. The paper is organized so it documents the development stages:

1. Definition of a system model, and specification of requirements.

* This work is partially funded by the Commission of the European Communities (CEC) under the ESPRIT programme in the field of Basic Research Project No. 7071: ProCoS: Provably Correct Systems".
2. Specification of a control design for the system and assumptions about the environment.

3. Verification of the control design by proving that the design refines the requirements under the assumptions.

Although the documentation is presented in a topdown fashion, it must be noted that development of a sufficiently abstract system model and a correct design is an iterative process.

In the conclusion we report experience with a proof assistant tool encoded in PVS $[8,10]$ for mechanical verification. In order to give a feeling of how proofs in the Duration Calculus are structured the detailed verifications are given in an appendix.

\section{Air Traffic Monitoring System}

The air traffic monitoring system monitors the position of civil aircraft in the vicinity of an airport. Aircraft may be landing at, departing from or crossing over the airport. The aims of the system are:

1. to ensure aircraft do not risk mid-air collision by coming too close to each other,

2. to organize landing and departings of aircraft, and

3. to collect position information from radar

The components of a system model are: an Airspace database containing the aircraft, a Radar that measures the positions of aircraft, an Alert mechanism that alerts aircraft in collision risk, and finally one or more Runways for the landing and departures of aircraft. The task of a software design for the system is to take care of the correct interplay between the components.

The tasks of the system motivates a modular composition of the overall system. To keep the presentation short, we will focus on the collision avoidance part of the system, and hence describe the interplay between Airspace and Alert components. This subsystem will be denoted as CAS, Collision Avoidance System. It should be noted that the developed model is kept deliberately simple. For instance the velocity of aircraft is not modeled, but this can easily be added, and is used in the larger model presented in [6]. 


\section{System Model}

In this section a system model of the CAS subsystem is derived in order to capture the safety requirement. First we introduce some design parameters:

$$
\begin{aligned}
& v_{\max }, r, R: \mathbb{R} \\
& T, T_{a l}, T_{\text {coll }}, T_{\text {init }}: \text { Time }
\end{aligned}
$$

These parameters will be explained below.

\section{Air space:}

A state is needed to track all aircraft in the controlled air space. This state, which is a real-time database recording all aircraft positions, will be named Pos. It is declared in a schema.

$$
\text { AIRSPACE }
$$$$
\text { Pos }: I D \rightarrow(\text { State POSITION })
$$

Since the notion of time is implicit in our notation we use the keyword State to denote time varying states. A variable with this keyword is interpreted as a function of time.

The database is a function from aircraft-identifiers, $[I D]$, to information about the aircraft, in this simple case a position, which changes over time. The fact that no aircraft can be in different places in same time is thus captured by the model variable. The positions are Cartesian coordinates with the origin being the location of the radar.

$$
\text { POSITION } \hat{=}[x: \mathbb{R} ; y: \mathbb{R} ; z: \mathbb{R}]
$$

Distance between positions is computed by a function dist. We define first a predicate Close, which holds if the distance between two aircraft becomes less than some input parameter $x$ ?:

\begin{tabular}{|l} 
Close \\
AIRSPACE \\
$x ?: \mathbb{R}$
\end{tabular}

Note that in $\mathrm{Z}$ all free variables are universally quantified.

Aircraft are surrounded and protected by a sphere, with radius, $r \geq 0$, that no other aircraft is permitted to enter. For simplicity we assume all aircraft have the same speed, and hence the same protection sphere. A violation of this sphere, i.e if the distance between two aircraft becomes less than $2 r$ is tantamount to a collision. Hence the predicate Coll:

$$
\text { Coll } \hat{=} \text { Close }[2 r / x ?]
$$

The predicate is defined by renaming of the input parameter $x$ ? to $r$.

\subsection{Requirements}

We consider a single safety requirement, $R E Q$, namely the fact that no collisions may take place in the controlled air space.

$$
R E Q \widehat{=} \square(\neg \text { Coll })
$$

The modal operator, $\square$, means for any interval of time. The formula says that for any interval of time the predicate $\neg$ Coll holds.

\section{Control System Design}

In this section a refinement of the system model towards a design is presented. The design consists of some assumptions that should be satisfied by the environment and a controller in terms of a finite state automaton.

\subsection{Assumptions}

To detect collision risks a bigger sphere with radius, $R>r$, surrounding each aircraft is needed. A collision risk appears when the distance between two arbitrary aircraft becomes less than $2 R$. Figure 1 illustrates the idea.

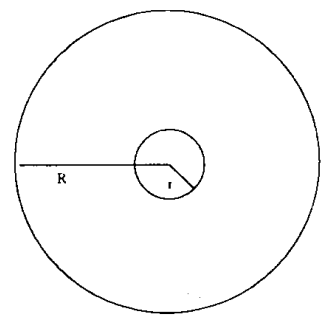

Figure 1: Protection spheres

We will also assume a general maximum velocity, $v_{\max }>0$, for all aircraft in the air space. The worst collision time, $T_{\text {coll }}$ occurs when two aircraft, separated by a distance of $2 R$, move against each other with this maximum velocity. This time is given by the equation

$$
T_{\text {coll }}=\frac{R-r}{v_{\max }}
$$

Furthermore we will assume that the positions only change after $T$ time units, i.e. a measured position is stable for time $T$. In order to be able to detect collision risks in time we require that the aircraft do not move too fast. These two assumptions are expressed as:

$$
\begin{aligned}
& A S M_{0} \\
& A I R S P A C E \square\left(\left\lceil P o s(i d) \neq p_{1}\right\rceil ;\left\lceil P o s(i d)=p_{1}\right\rceil ;\right. \\
&\left.\left\lceil P o s(i d) \neq p_{1}\right\rceil \Rightarrow \ell>T\right) \\
& \square\left(\left\lceil P o s(i d)=p_{1}\right\rceil ; \operatorname{true} ;\left\lceil P o s(i d)=p_{2}\right\rceil\right. \\
&\left.\Rightarrow \operatorname{dist}\left(p_{1}, p_{2}\right) \leq v_{\max } \cdot(\ell+T)\right) \\
& \hline
\end{aligned}
$$


These formula use the chop operator ' $;$ ' to divide an interval into contiguous subintervals. The first formula considers an interval, where a given aircraft id change to position $p_{1}$ and then away again. For any interval of this form the length, $\ell$, is greater than $T$. The second formula states that for any interval consisting of an initial subinterval where an aircraft with id has position $p_{1}$ followed by a unspecified subinterval, true, and followed by a third subinterval in which the same aircraft has now position $p_{2}$ then the flown distance is less than $v_{\max } \cdot(\ell+T)$.

From $A S M_{0}$ we are able to set an upper bound on the sampling time $T$. First we need a predicate, AllSafe, which holds if all aircraft are safely separated:

$$
\text { AllSafe } \hat{=} \neg \text { Close }[2 R / x ?]
$$

Fact1: A potential collision is detectable if all aircraft are safe before a collision and if $T \leq T_{\text {coll }}$.

$$
\square\left(\text { AllSafe ; true ; Coll } \Rightarrow \ell \geq T_{\text {coll }}-T\right)
$$

Fact2: A collision risk may be missed by the system if $T>T_{\text {coll }}$.

$$
\square\left(\text { AllSafe } ; \text { Coll } \Rightarrow T>T_{\text {coll }}\right)
$$

Proofs of these facts are given in Appendix B. As a consequence of these lemmas we will assume that $T_{\text {coll }} \geq T$.

Alert:

To ensure safety, the system must alert the pilots of aircraft being in a collision risk, i.e. whenever two aircraft come too close to each other. The pilots of aircraft in collision are then alerted via an air traffic controller. We introduce a state variable, Alert, a set of identifiers, for aircraft in collision risk. The states of the CAS system are now collected in the schema:

$$
\begin{aligned}
& \text { CAS_STATES } \\
& \text { AIRSPACE } \\
& \text { Alert : State P ID }
\end{aligned}
$$

The interplay between Alert and the pilots is not specified in this work, but instead we assume that an intelligent plan for avoiding the collisions is produced by the air traffic controller, and that the pilots follow the plan and are out of the danger zone within $T_{a l}$ time units. Finally we will assume that in an initial interval of length $T_{\text {init }}$ all aircraft are safely separated. The above assumptions are now formalised in a schema:

$\left[\begin{array}{l}A S M_{1} \\ C A S_{-} S T A T E S \\ A S M_{0} \\ \hline R>r \\ T_{\text {coll }} \geq T \\ \lceil\text { InDanger } \subseteq \text { Alert }\rceil \stackrel{T_{q l}}{\rightarrow}\lceil\text { InDanger }=\{\}\rceil \\ 0<\ell \leq T_{\text {init }} \Rightarrow \text { AllSafe } \\ \hline\end{array}\right.$

The third assumption is expressed by use of the timed progress operator:

$$
\begin{aligned}
D_{1} \stackrel{T}{\rightarrow} D_{2} \hat{=} \square\left(\left(D_{1} \wedge \ell=T\right) ; \ell>0 \Rightarrow\right. \\
\left.\ell=T ; D_{2} ; \text { true }\right)
\end{aligned}
$$

The formula states that if a formula $D_{1}$ holds for time $T$ on an interval then formula $D_{2}$ holds initially on a succeeding non-point interval.

The function InDanger finds all aircraft being in a collision risk.

\begin{tabular}{|l} 
InDanger \\
AIRSPACE \\
$d s !:$ State PID \\
\hline$d s !=(\lambda t:$ Time $\bullet$ \\
$\quad\{i d \mid i d: \operatorname{dom}$ Pos $\wedge$ \\
$\quad \exists i d_{1}: \operatorname{dom}$ Pos $\bullet$ id $\neq i d_{1} \wedge$ \\
$\left.\left.\quad \operatorname{dist}\left(\operatorname{Pos}(i d)(t), \operatorname{Pos}\left(i d_{1}\right)(t)\right)<2 R\right\}\right)$ \\
\hline
\end{tabular}

The output parameter, $d s$ !, records the aircraft having a collision risk as a function of time.

\subsection{Controller Design}

The controller design is based on a finite state automaton. We introduce a new type PHASES to represent the phases of the automaton.

\section{[PHASES $]$}

The control automaton consists of three different phases. These are introduced as:

$$
\mid \begin{aligned}
& \text { Init, Idle, Act }: \text { PHASES } \\
& \text { Init } \neq \text { Idle } \wedge \text { Idle } \neq \text { Act } \wedge \text { Act } \neq \text { Init }
\end{aligned}
$$

The Init phase describes the initial behaviour of the controller. In the Idle phase the automaton does nothing, and in the Act phase the automaton performs actions for avoiding possible collisions. The abbreviations [Init], [Idle] and [Act] are in the following used to denote the current phase of the automaton. The phases of the automaton and the transitions between them are depicted in figure 2.

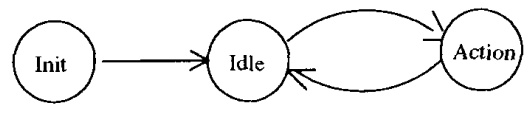

Figure 2: automaton

We need two new constants: $\epsilon$ and $\delta$. The parameter $\epsilon$ is used to specify a latency time for the system to react and move from one phase to another. The parameter $\delta$ is used for a latency time for the system to enable the alert mechanism.

$$
\begin{aligned}
& \epsilon, \delta: \text { Time } \\
& T_{\text {coll }} \geq T+T_{a l}+\delta+\epsilon
\end{aligned}
$$


We decide that the automaton starts in the Init phase, and that the Init phase is followed by the Idle phase within $T_{\text {init }}$ time units.

$$
\operatorname{INITIAL} \hat{=}(\lceil\rceil \rightarrow\lceil\text { Init }\rceil) \wedge\left(\lceil\text { Init }\rceil \stackrel{T_{\text {init }}}{\rightarrow}\lceil\text { Idle }\rceil\right)
$$

The next schema states that the Idle phase is followed by the Act phase.

$$
T R A N S \hat{=}\lceil\text { Idle }\rceil \rightarrow\lceil\text { Act }\rceil
$$

The predicate Danger, defined as

$$
\text { Danger } \hat{=} \text { Close[2R/x?] }
$$

determines when a transition is allowed to be performed. The predicate states simply if there is risk for a collision. The enabled transitions are specified in the schema ENAB_TRANS.

\begin{tabular}{l} 
ENAB_TRANS \\
AIRSPACE \\
\hline$\lceil$ Idle $\rceil \wedge$ Danger $\stackrel{\epsilon}{\rightarrow}\lceil$ Act $\rceil$ \\
$\lceil$ Act $\rceil \wedge \neg$ Danger $\stackrel{\epsilon}{\rightarrow}\lceil$ Idle $\rceil$ \\
\hline
\end{tabular}

The next schema, CON_STABILITY, describe the conditions needed to maintain a phase.

\begin{tabular}{l}
$C O N_{-S T A B I L I T Y}$ \\
AIRSPACE \\
\hline$\lceil$ Idle $\rceil \wedge \neg$ Danger $\rightarrow\lceil$ Idle $\rceil$ \\
$\lceil$ Act $\rceil \wedge$ Danger $\rightarrow\lceil$ Act $\rceil$ \\
\hline
\end{tabular}

The actuator part of the system, i.e. the alert mechanism, is activated in the Act phase. This decision is specified as:

\begin{tabular}{|l} 
ACTIONS \\
\hline CAS_STATES \\
$\lceil$ Act $\rceil \stackrel{\delta}{\rightarrow}\lceil$ InDanger $\subseteq$ Alert $\rceil$ \\
\hline
\end{tabular}

The total specification of the controller design is given by a collection of schemas:

$$
\begin{aligned}
D E S_{1} \hat{=} & I N I T I A L \wedge \text { TRANS } \\
& \text { ENAB_TRANS } \wedge \text { ACTIONS } \\
& \text { CON_STABILITY }
\end{aligned}
$$

As a final specification we introduce a schema for the control design of the CAS subsystem:

$$
C A S_{1} \hat{=} D E S_{1} \wedge A S M_{1}
$$

The proof obligation is then to show the correctness of the control design, i.e.

$$
C A S_{1} \Rightarrow R E Q
$$

which is verified in Appendix A.

\section{Conclusion}

We have illustrated an application of a Timed-Z notation comprising of an embedding of the Duration Calculus into the $Z$ specification language. The advantages of this is twofold: besides specifying and reasoning about timing properties of real-time systems, it provides also composite modular specifications. The latter is a necessity when dealing with complex systems. The example of the specification of a subsystem of the Air Traffic Monitoring System illustrates this thesis. The other subsystems have been specified in a similar manner as done here [6].

Another advantage of the presented approach is the possibility for application of the existing software tools. The presented specification and verification has been checked by the DC/PC tool. This has resulted in detection of some missing details. For instance the stability of the measured positions was not captured in the initial specification, but the proof assistant insisted on the necessity of this fact. Thus for large scale development of real-time systems the assistance of tools for type checking and mechanical verification is indispensable. This will not reduce design time, but it will certainly liberate the designer from the tedious parts of the verification, and ensure the correctness of the design.

\section{Acknowledgements}

I am very grateful to Anders P. Ravn for his inspiring and indeed very valuable comments and ideas on this case study. Thanks to Kirsten M. Hansen and Jens U. Skakkebæk for their helpful comments and suggestions.

\section{References}

[1] D. Bjørner. A ProCoS Project Description. ESPRIT BRA 3104, EATCS Bull. no. 39, Oct. 1989.

[2] S.M. Brien, M. Engel, He Jifeng, H. Rischel, and A.P. Ravn. Z Description of Duration Calculus. Technical report, Draft. OU HJF 12/2

[3] J. Bowen, M. Franzle, E.R. Rüdiger, and A.P. Ravn. Developing Correct Systems. Proc. 5th Euromicro Workshop on Real-Time Systems, pages 176-187, June 1993.

[4] M. Engel. Specifying Real-Time Systems with Z and the Duration Calculus. To appear in $Z U W^{\prime} 94$

[5] K.M. Hansen, H.Rischel, and A.P. Ravn. Specifying and Verifying Requirements of Real-Time Systems. IEEE Transactions on Software Engineering, Vol 19(1):41-55, 1993.

[6] R. Inal. Specifying and Verifying Real-Time Systems. Master's thesis, ID/DTH/RI, 1993.

[7] K. Jackson. Aircraft Traffic Control. Technical report, University of Stirling, 1992. 
[8] S. Owre, N. Shankar, and J.M. Rushby. User Guide for the PVS Specification and Verification System, Language and Proof Checker. Technical report, CSL, SRI Intl., October 1993.

[9] J.U. Skakkebæk, H. Rischel, A.P. Ravn, and Z. Chaochen. Specification of Embedded Real-Time Systems. Proc. 4th Euromicro Workshop on RealTime Systems, pages 116-121, June 1992.

[10] J.U. Skakkebæk, and N. Shankar. Towards a Duration Calculus Proof Assistant in PVS. Technical Report ID/DTH/JUS 5/1, Lyngby, Denmark March 1994. Paper version submitted for publication.

[11] J.M. Spivey. The Z Notation: A Reference Manual. Prentice-Hall, 2nd edition, 1992.

\section{A Appendix A}

Our single safety requirement is proved by contradiction. Assume

$$
\neg R E Q \Leftrightarrow \neg(\square(\neg \text { Coll })) \Leftrightarrow \diamond \text { Coll }
$$

The formula, $\diamond$ Coll, is split into three cases:

Case a : Coll; true

Case b : AllSafe ; Coll

Case c: AllSafe ; Danger ; Coll

which corresponds to occurrence of a collision, respectively, at the beginning of the observation interval, immediately after a situation, where all aircraft are safely separated, and immediately after a situation, where a collision risk is observed.

Case b can not occur, because our design does not, by Fact 2 and $A S M_{1}$, allow such big changes of positions. We examine now the first case. INITIAL decomposes to [ ] or [Init $\rceil$; true. It is easy to see that \lceil\rceil$\wedge \mathrm{Coll} ;$ true $\Rightarrow$ false. The rest of the analysis of the first case gives:

$$
\begin{aligned}
& \text { Coll; true } \wedge \text { [Init }\rceil ; \text { true } \\
& \Rightarrow\{I N I T I L\} \\
& \text { Coll ; true } \wedge\left(\ell \leq T_{\text {init }} \wedge\lceil\text { Init }\rceil \vee\right. \\
& \left.\quad \ell=T_{\text {init }} ;\lceil\text { Idle }\rceil ; \text { true }\right) \\
& \Rightarrow\left\{P \text { Logic, } A S M_{1}\right\} \\
& \text { Coll } ; \text { true } \wedge \text { AllSafe } \vee \\
& \text { Coll } ; \text { true } \wedge \text { AllSafe; }\lceil\text { Idle }\rceil ; \text { true } \\
& \Rightarrow\{D C\} \\
& \diamond(\text { Coll } \wedge \text { AllSafe }) \\
& \Rightarrow\{R>r\} \\
& \diamond \text { false } \\
& \Rightarrow\{D C\} \\
& \text { false }
\end{aligned}
$$

Before starting on the analysis of Case $\mathrm{c}$ we state a lemma:

Lemma1: If the automaton is in the Idle phase while there is danger for collision then the danger will be avoided within $\epsilon+\delta+T_{a l}$ time units.

$$
\begin{aligned}
& \square(([\text { Idle }\rceil ; \text { true } \wedge \text { Danger }) \Rightarrow \\
& \left.\quad\left(\ell \leq \epsilon+\delta+T_{a l} \vee \ell=\epsilon+\delta+T_{a l} ; \text { AllSafe }\right)\right)
\end{aligned}
$$

Proof:

$$
\begin{aligned}
& \text { [Idle }] \text {; true } \wedge \text { Danger } \\
& \Rightarrow\left\{E N A B_{-} T R A N S\right\} \\
& \vec{\ell} \leq \epsilon \wedge[\text { Idle }] \wedge \text { Danger } \vee \\
& \ell=\epsilon ;(\lceil\text { Act }\rceil ; \text { true } \wedge \text { Danger }) \\
& \Rightarrow\{\ell \geq \epsilon\} \\
& \ell=\epsilon ;([\text { Act }] ; \text { true } \wedge \text { Danger }) \\
& \Rightarrow\{A C T I O N S, \ell \geq \epsilon+\delta\} \\
& \ell=\epsilon+\delta ;([\text { Act }] ; \text { true } \wedge \text { Danger } \wedge \\
& \lceil\text { InDanger } \subseteq \text { Alert }\rceil) \\
& \Rightarrow\left\{A S M_{1}, C O N_{-} S T A \bar{B} I L I T Y, \ell \geq \epsilon+\delta+T_{a l}\right\} \\
& \ell=\epsilon+\delta+T_{a l} ;(\lceil\text { Act }\rceil ; \text { true } \wedge\lceil\text { InDanger }=\emptyset\rceil) \\
& \Rightarrow\{\text { Definitions }\} \\
& \ell=\epsilon+\delta+T_{a l} ; \text { AllSafe }
\end{aligned}
$$

Case is split into three more cases, each depending on the current phase of the automaton. We will go through the case, where the current phase is Idle:

$$
\begin{aligned}
& \text { 「Idle }\rceil \text {; true } \wedge \text { (AllSafe; Danger ; Coll }) \\
& \Rightarrow\left\{C O N \_S T A B I L I T Y\right\} \\
& \text { AllSafe ; ([Idle }\rceil \text {; true } \wedge \text { Danger ; Coll }) \\
& \Rightarrow\{\text { Lemma1 }\} \\
& \text { AllSafe } ;\left(\ell \leq \epsilon+\delta+T_{a l} \vee\right. \\
& \Rightarrow\{D C\} \\
& \left.\left.\ell=\epsilon+\delta+T_{a l} ; \text { AllSafe }\right) ; \text { Coll }\right) \\
& \text { AllSafe } ; \ell \leq \epsilon+\delta+T_{a l} ; \text { Coll } \vee \\
& \text { AllSafe } ; \ell=\epsilon+\delta+T_{a l} ; \text { AllSafe } ; \text { Coll } \\
& \Rightarrow\left\{\text { Fact } 1, \ell>\frac{R-r}{v_{m a}}-T \geq \epsilon+\delta+T_{a l}\right\} \\
& \text { false } \vee \text { AllSafe; Coll } \\
& \Rightarrow\{\text { Case b, PLogic }\} \\
& \text { false }
\end{aligned}
$$

The implication in the last case has been derived under the assumption

$$
\ell>\frac{R-r}{v_{\max }}-T \geq \epsilon+\delta+T_{a l}
$$

Hence the final general design decision is:

$$
R-r \geq\left(T+T_{a l}+\delta+\epsilon\right) \cdot v_{\max }
$$

This completes the verification of the correctness of the control design.

\section{B Appendix B}

Fact1: A potential collision is detectable if all aircraft are safe before a collision and if $T \leq T_{\text {coll }}$.

$$
\square\left(\text { AllSafe ; true ; Coll } \Rightarrow \ell \geq T_{\text {coll }}-T\right)
$$


Proof:

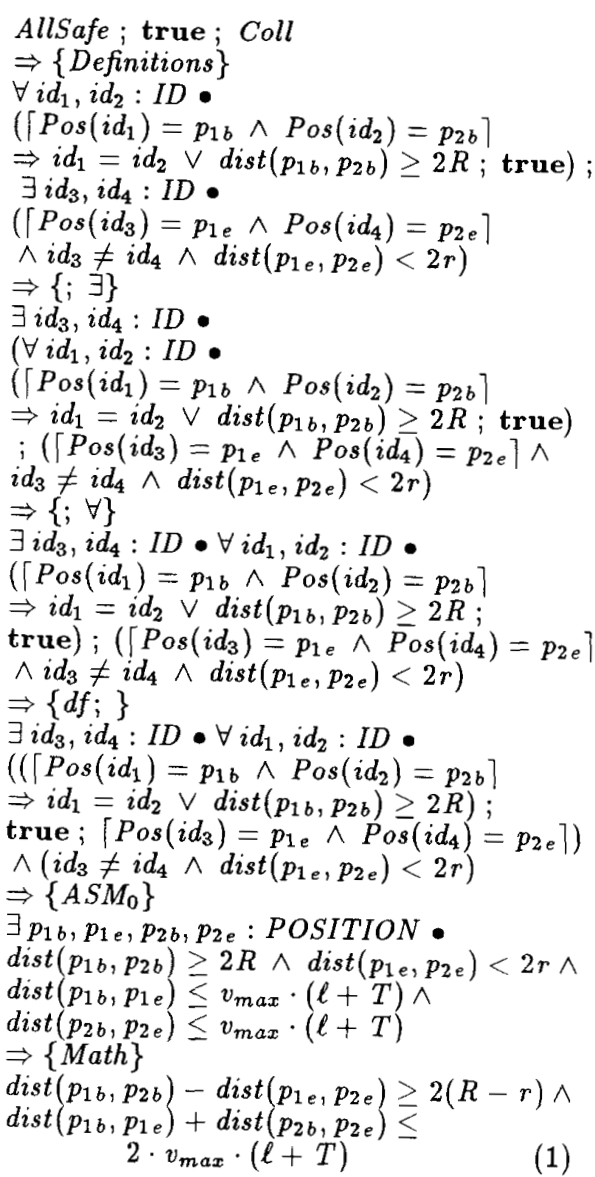

Now we will use some properties of the function dist. The triangle inequality applied twice gives

$$
\begin{aligned}
& \operatorname{dist}\left(p_{1 b}, p_{1 e}\right)+\operatorname{dist}\left(p_{1 e}, p_{2 e}\right)+\operatorname{dist}\left(p_{2 e}, p_{2 b}\right) \geq \\
& \quad \operatorname{dist}\left(p_{1 b}, p_{2 b}\right) \\
& \Rightarrow\{\text { Symmetry, Arithmetic }\} \\
& \operatorname{dist}\left(p_{1 b}, p_{1 e}\right)+\operatorname{dist}\left(p_{2 b}, p_{2 e}\right) \geq \\
& \Rightarrow\{(1)\} \quad \operatorname{dist}\left(p_{1 b}, p_{2 b}\right)-\operatorname{dist}\left(p_{1 e}, p_{2 e}\right) \\
& 2 \cdot v_{\max } \cdot(\ell+T) \geq 2(R-r) \\
& \Rightarrow\{\text { Math }\} \\
& \ell \geq \frac{R-r}{v_{m a x}}-T
\end{aligned}
$$

In order to have $\ell \geq 0$ we choose the sampling time such that $T \leq \frac{R-r}{v_{\max }}=T_{\text {coll }}$.

Fact 2 is proved in a similar way. 